\title{
The Face-Centred Finite Volume Methods for Incompressible Flows
}

\author{
Luan M. Vieira ${ }^{*, \dagger,+}$, Matteo Giacomini ${ }^{\dagger, *}$, Rubén Sevilla $^{+}$and Antonio Huerta ${ }^{\dagger, *}$ \\ * International Center for Numerical Methods in Engineering (CIMNE) \\ e-mail: lmalikoski@cimne.upc.edu \\ † Laboratori de Càlcul Numèric (LaCàN), ETS de Ingenieros de Caminos, Canales y Puertos, \\ Universitat Politècnica de Catalunya \\ Campus Norte UPC, 08034 Barcelona, Spain \\ + Zienkiewicz Centre for Computational Engineering, Faculty of Science and Engineering, \\ Swansea University, \\ Bay Campus, SA1 8EN, Wales, United Kingdom
}

\begin{abstract}
The Face-Centred finite volume (FCFV), which stems from a mixed-formulation of the Discontinuous Galerkin (DG) methods, is presented for the incompressible Navier-Stokes equations. The main feature of the FCFV formulation is the definition of the solution at the centre of cell faces. In contrast, existing Cell-Centred (CCFV) and Vertex-Centred (VCFV) finite volume formulations available in many CFD require the reconstruction of gradients at the cell faces. As a consequence, those technologies experience suboptimal convergence when the grid is distorted or stretched [4-6]. On the contrary, the FCFV achieves first-order accuracy for the solution and its gradient without the need of flux reconstruction. Hence, FCFV preserves its accuracy irrespective of grid stretching or distortion [1-3]. From an industrial perspective, this flexibility in mesh generation is a critical aspect in face of the complex geometric configurations to be studied in CFD problems. Furthermore, the FCFV formulation naturally circumvents the need for pressure correction strategies to deal with the velocity-pressure coupling in incompressible flow problems. The capabilities of the FCFV for the simulation of incompressible Navier-Stokes equations will be presented via numerical experiments involving both academic and industrial benchmarks.
\end{abstract}

\section{REFERENCES}

[1] Sevilla, R. and Giacomini, M. and Huerta, A. A face-centred finite volume method for secondorder elliptic problems.Int. J. Num. Meth. in Engng, Vol. 115, pp. 986-1014, (2018)

[2] Vieira, L.M. and Giacomini, M. and Sevilla, R. and Huerta,A . A second-order face-centred finite volume method for elliptic problems. CMAME, Vol. 358, pp. 112655, (2020)

[3] Giacomini, M. and Sevilla, R. A second-order face-centred finite volume method on general meshes with automatic mesh adaptation. Int. J. Num. Meth. in Engng, Vol. 121, pp. 5227$5255,(2020)$

[4] D. McBride and N. Croft and M. Cross, Finite volume method for the solution o flow on distorted meshes. Int. J. Num. Meth. for heat and fluid flow, Vol 17, pp. 213-239, (2007)

[5] Diskin, B. and Thomas, J.L.Notes on accuracy of finite-volume discretization schemes in irregular grids.. Applied Numerical Mathematics. Vol 60, pp. 224-226, (2010)

[6] Wang, N. and Li, M. and Ma, R. and Zhang, L. Accuracy analysis of gradient reconstruction on isotropic unstructured meshes and its effects on iniviscid flow simulations. Advances in Aerodynamics, Vol. 1, pp. 1-31, (2019) 